\title{
Second and third trimester fetal ultrasound population screening for risks of preterm birth and small-size and large-size for gestational age at birth: a population-based prospective cohort study
}

\section{Fetal ultrasound screening for common adverse birth outcomes}

Jan S. Erkamp ${ }^{1,2}$, Ellis Voerman ${ }^{1,2}$, Eric A. P. Steegers ${ }^{3}$, Annemarie G. M. G. J. Mulders ${ }^{3}$, Irwin K. M. Reiss ${ }^{1,4}$, Liesbeth Duijts ${ }^{1,4,5}$, Vincent W. V. Jaddoe ${ }^{1,2}$ and Romy Gaillard ${ }^{1,2^{*}}$

\begin{abstract}
Background: Preterm birth, small size for gestational age (SGA) and large size for gestational age (LGA) at birth are major risk factors for neonatal and long-term morbidity and mortality. It is unclear which periods of pregnancy are optimal for ultrasound screening to identify fetuses at risk of preterm birth, SGA or LGA at birth. We aimed to examine whether single or combined second and third trimester ultrasound in addition to maternal characteristics at the start of pregnancy are optimal to detect fetuses at risk for preterm birth, SGA and LGA.

Methods: In a prospective population-based cohort among 7677 pregnant women, we measured second and third trimester estimated fetal weight (EFW), and uterine artery pulsatility and umbilical artery resistance indices as placenta flow measures. Screen positive was considered as EFW or placenta flow measure $<10$ th or $>90$ th percentile. Information about maternal age, body mass index, ethnicity, parity, smoking, fetal sex and birth outcomes was available from questionnaires and medical records. Screening performance was assessed via receiver operating characteristic (ROC) curves and area under the curve (AUC) along with sensitivity at different false-positive rates.

\footnotetext{
* Correspondence: r.gaillard@erasmusmc.nl

'The Generation R Study Group, Erasmus MC, University Medical Center Rotterdam, P.O. Box 2040, 3000, CA, Rotterdam, the Netherlands

${ }^{2}$ Department of Paediatrics, Erasmus MC, University Medical Center Rotterdam, Rotterdam, The Netherlands

Full list of author information is available at the end of the article
}

(c) The Author(s). 2020 Open Access This article is licensed under a Creative Commons Attribution 4.0 International License, which permits use, sharing, adaptation, distribution and reproduction in any medium or format, as long as you give appropriate credit to the original author(s) and the source, provide a link to the Creative Commons licence, and indicate if changes were made. The images or other third party material in this article are included in the article's Creative Commons licence, unless indicated otherwise in a credit line to the material. If material is not included in the article's Creative Commons licence and your intended use is not permitted by statutory regulation or exceeds the permitted use, you will need to obtain permission directly from the copyright holder. To view a copy of this licence, visit http://creativecommons.org/licenses/by/4.0/ The Creative Commons Public Domain Dedication waiver (http://creativecommons.org/publicdomain/zero/1.0/) applies to the data made available in this article, unless otherwise stated in a credit line to the data. 


\begin{abstract}
(Continued from previous page)
Results: Maternal characteristics only and in combination with second trimester EFW had a moderate performance for screening for each adverse birth outcome. Screening performance improved by adding third trimester EFW to the maternal characteristics (AUCs for preterm birth 0.64 (95\%Cl 0.61 to 0.67$)$; SGA 0.79 ( $95 \% \mathrm{Cl} 0.78$ to 0.81 ); LGA $0.76(95 \% \mathrm{Cl} 0.75 ; 0.78)$ ). Adding third trimester placenta measures to this model improved only screening for risk of preterm birth (AUC $0.72(95 \% \mathrm{Cl} 0.66$ to 0.77$)$ with sensitivity $37 \%$ at specificity $90 \%)$ and SGA (AUC $0.83(95 \% \mathrm{Cl} 0.81$ to 0.86 ) with sensitivity $55 \%$ at specificity $90 \%$ ). Combining second and third trimester fetal and placental ultrasound did not lead to a better performance as compared to using only third trimester results.

Conclusions: Combining single third trimester fetal and placental ultrasound results with maternal characteristics has the best screening performance for risks of preterm birth, SGA and LGA. As compared to second trimester screening, third trimester screening may double the detection of fetuses at risk of common adverse birth outcomes.
\end{abstract}

Keywords: Fetal growth, Preterm birth, Small size for gestational age, Large size for gestational age, Cohort study, Screening, Third trimester ultrasound

\section{Background}

Preterm birth, small size for gestational age (SGA) and large size for gestational age (LGA) at birth explain up to $30 \%$ of neonatal death and are strong risk factors for short-term and long-term morbidity [1, 2]. The majority of newborns who experience abnormal fetal growth are unidentified until birth [3-6]. SGA or LGA newborns who have not been identified antenatally have strongly increased risks of morbidity and mortality, compared to those who have been identified antenatally [6-9]. Abnormal fetal growth is an important reason for induction of labour and is therefore a common cause of induced preterm birth $[3,10]$. However, studies have shown that spontaneous preterm birth is often preceded by impaired or accelerated fetal growth [3, 9]. Current pregnancy care protocols include dating ultrasounds and detailed structural ultrasounds at 20 weeks gestational age (GA) to assess congenital anomalies and fetal size [11, 12]. Third trimester ultrasound screening is mostly used in selected populations. Technological developments in obstetric ultrasounds may lead to future changes in ultrasound screening protocols, such as early-pregnancy size and congenital anomaly assessment and third trimester growth assessment. The performance of routine third trimester ultrasound screening, independent of other maternal and fetal characteristics, is not clear. A review of eight controlled trials did not suggest consistent benefits of ultrasound after 24 weeks GA on pregnancy outcomes [13]. A prospective observational cohort study among 3977 nulliparous women suggested that third trimester ultrasound, in addition to second trimester ultrasound, tripled the detection of fetuses subsequently born SGA compared to selective third trimester ultrasound [14].

We used data from a population-based observational study among 7670 pregnant women to examine whether single or combined second or third trimester fetal and placental ultrasound examinations, in addition to maternal characteristics, are optimal to detect fetuses at risk for preterm birth, SGA and LGA.

\section{Methods \\ Study design}

This study was embedded in the Generation R Study, a population-based prospective cohort study from early pregnancy onwards in Rotterdam, the Netherlands [15]. The study has been approved by the local Medical Ethical Committee (MEC 198.782/2001/31). Written consent was obtained from all women. All pregnant women were enrolled between 2001 and 2005. The response rate at birth was $61 \%$, which was calculated by dividing the number of participating live born children by the total number of live born children born in the study area during the inclusion period [16]. A total of 8879 women were enrolled during pregnancy. We excluded women without second and third trimester ultrasound data $(n=$ 1130), non-singleton live-births $(n=33)$, and women with outcome data missing $(n=46)$. This led to a population for analysis of 7670 pregnant women (Additional file 1, Figure S1 shows the flowchart for the population for analysis). Additional file 2 contains a Strengthening the Reporting of Observational Studies in Epidemiology (STROBE) statement for the current study [17].

\section{Maternal characteristics at the start of pregnancy}

We selected maternal characteristics known at the start of pregnancy, which are important determinants of adverse birth outcomes [3, 18-20]. Maternal age was assessed at enrolment and categorized; < 25.0 years, 25.0-34.9 years, $\geq 35.0$ years [3]. Maternal height $(\mathrm{cm})$ and weight $(\mathrm{kg})$ were measured without shoes and heavy clothing at enrolment and BMI $\left(\mathrm{kg} / \mathrm{m}^{2}\right)$ was calculated 
and categorized for clinical purposes: normal weight (BMI $<25 \mathrm{~kg} / \mathrm{m}^{2}$ ), overweight (BMI $25.0-30.0 \mathrm{~kg} / \mathrm{m}^{2}$ ) and obese $\left(B M I \geq 30.0 \mathrm{~kg} / \mathrm{m}^{2}\right)$ [19]. Information about ethnicity and parity and smoking was obtained by questionnaire and categorized as previously described $[3,18]$.

\section{Second and third trimester fetal and placental ultrasounds}

Ultrasound examinations were carried out in two dedicated research centres in first (median 13.2 weeks GA, interquartile range (IQR) 12.2 to 14.7), second (median 20.5 weeks GA, IQR 19.9 to 21.3) and third trimester (median 30.4 weeks GA, IQR 29.8 to 30.9) [3]. We established GA by using data from the first ultrasound [3]. In second and third trimesters, we measured fetal head circumference, abdominal circumference (AC) and femur length to the nearest millimeter using standardized procedures [21]. Estimated fetal weight (EFW) was calculated using the formula of Hadlock et al., in line with clinical practice [22]. GA-adjusted SDS for growth measures were based on reference growth charts from the whole study population [3]. In line with clinical practice, we defined screen-positive as EFW or AC in the lowest or highest decile in second or third trimester $[5,8,14$, 23, 24]. Both extremes of EFW and AC are associated with a higher risk of common adverse birth outcomes and perinatal morbidity and mortality [3, 14]. This approach leads to one screening test for all adverse birth outcomes, which strongly improves ease-of-use in clinical practice. However, EFW > 90th percentile is not associated with an increased risk of delivering a SGA newborn. Similarly, EFW < 10th percentile is not associated with an increased risk of delivering a LGA newborn. Thus, defining screen positive as EFW < 10th percentile and $>90$ th percentile for all adverse birth outcomes in our screening models may reduce the observed screening performance. The performance of the screening model may be improved when we define screen positive separately for SGA (as EFW < 10th percentile) and for LGA (as EFW > 90th percentile). We consider one combined screening test for all adverse birth outcomes more applicable for clinical practice, but to assess whether this affects the observed screening performance, we also evaluated screening performance of models in which we defined "screen-positive" separately for SGA $($ EFW < p10) and LGA (EFW > p90). Second-to-third trimester EFW or AC change was classified screen-positive if the change was in the lowest or highest decile.

Uterine artery resistance indices (UtA-RI) and umbilical artery pulsatility indices (UA-PI) are measures of vascular resistance in the uterine and umbilical arteries, respectively. Increased UtA-RI and UA-PI are associated with impaired placental vascular development and increased risks of abnormal intrauterine growth and adverse perinatal outcomes [23, 25-29]. These parameters may therefore be of additional value in clinical screening models. These parameters were derived from flow velocity waveforms in second and third trimesters [30]. We defined screenpositive UtA-RI or UA-PI or second-to-third trimester change as a value in the highest decile.

\section{Birth outcomes}

Information about offspring sex, GA and weight at birth, gestational hypertensive disorders, assisted vaginal delivery and cesarean delivery was obtained from medical records [15]. GA-adjusted SDS for birth weight was constructed using North European growth standards [31]. Preterm birth was defined as GA $<37$ weeks at birth. Spontaneous preterm birth was defined as spontaneous preterm labour or preterm premature rupture of membranes resulting in birth $<37$ weeks' GA. According to clinical standards, SGA and LGA at birth were defined as a GA-adjusted birth weight < 10th and > 90th percentile in the study cohort, respectively.

\section{Statistical analyses}

First, we calculated the absolute percentages of screen positive second and third trimester fetal ultrasounds among newborns born preterm, SGA and LGA. Second, we aimed to assess screening performance for preterm birth, SGA and LGA based on different predefined screening models. We constructed five predefined logistic regression models for screening of preterm birth, SGA and LGA, respectively. Preterm birth, SGA and LGA were the dependent variables in these different predefined logistic regression models. For each logistic regression model, we assessed the variance explained of the model. We obtained predicted values from these regression models and further assessed model performance via receiver operating characteristic (ROC) curves and calculation of the area under the curve (AUC), along with the sensitivity at different falsepositive rates (1-specificity). The five predefined logistic regression models for screening of preterm birth, SGA and LGA were as follows: (1) maternal characteristics model including maternal age, BMI, ethnicity, parity and smoking and fetal sex; (2) second trimester model (model 1 plus screening result based on second trimester EFW); (3) third trimester model (model 1 plus screening result based on third trimester EFW); (4) combined second and third trimester model (model 1 plus screening result based on second and third trimester EFW); (5) second-to-third trimester fetal growth model (model 4 plus second-tothird trimester EFW change). To compare model performance of the different predefined models, we assessed the change in effect size of the obtained AUCs from the different models. If the change in effect size was considered clinically relevant, we used the method by DeLong et al. for assessing whether the AUCs for two or more 
correlated receiver operating characteristic curves were statistically significantly different [32]. Positive and negative predictive values (PPV, NPV) and positive and negative likelihood ratios (PLR, NLR) at a 10\% false-positive rate ( $90 \%$ specificity) were calculated for our best model. Third, in a subsample of women with placenta flow measures available, we assessed the additional screening performance of placenta measures by adding second and third trimester UA-PI and UtA-RI screening results to the five models using a similar approach. To test the robustness of our findings, we performed 8 formal sensitivity analyses. We assessed (1) whether screening performance for spontaneous preterm birth was similar to screening performance for any preterm birth, (2) whether using stricter cut-off values to define screen-positive results improved screening performance $(E F W<5$ th percentile or EFW > 95th percentile), (3) whether our models improved when we used AC instead of EFW, (4) whether using only UA-PI or UtA-RI screening results leads to comparable screening performance as using both measurements combined, (6) whether defining "screen-positive" for individual outcomes separately (screen positive as EFW <10th percentile only for SGA and screen positive as EFW > 90th percentile only for LGA), instead of defining screenpositive as either EFW < 10th or EFW > 90th for all adverse birth outcomes, affects screening performance, (7) whether the screening performance changed when the outcome SGA was defined as moderate or extreme SGA (gestational-age-adjusted birth weight $<5$ th or $<3$ rd percentile, respectively) or defined as moderate or extreme LGA (gestational-age-adjusted birth weight $>95$ th or $>$ 97th percentile, respectively), (8) whether performance of our model was similar for selecting SGA or LGA newborns with adverse outcomes (SGA pregnancies complicated by gestational hypertensive disorders and LGA pregnancies resulting in delivery using assisted vaginal delivery or cesarean section). Finally, to assess how maternal characteristics affect our obtained screening performance of the different screening models, we assessed the screening performance of second and third trimester ultrasound without incorporating maternal characteristics in the models. To deal with missing values, we added a missing category for each maternal and fetal characteristic to the models. This approach resembles clinical practice. Analyses were performed using the Statistical Package of Social Sciences version 24.0 for Windows (IBM Corp., Armonk, NY, USA).

\section{Results}

\section{Participants characteristics}

Table 1 shows that 345 (4.5\%) newborns were born preterm, $768(10 \%)$ were SGA, and $767(10 \%)$ were LGA at birth. Additional file 1, Table S1 gives all fetal and
Table 1 Characteristics of mothers and their children $(N=7670)$

\begin{tabular}{|c|c|}
\hline Characteristics & Value $^{a}$ \\
\hline \multicolumn{2}{|l|}{ Maternal characteristics } \\
\hline Age, median (IQR), years & $30.3(25.9$ to 33.4$)$ \\
\hline$<25$, no. (\%) & $1573(20.5)$ \\
\hline $25-35$, no. $(\%)$ & $4972(64.8)$ \\
\hline$>35$, no. (\%) & $1125(14.7)$ \\
\hline Height, mean (SD) (cm) & $167.3(7.4)$ \\
\hline Weight, mean (SD) (kg) & $69.3(13.2)$ \\
\hline Body mass index ${ }^{1}$, mean $(\mathrm{SD})\left(\mathrm{kg} / \mathrm{m}^{2}\right)$ & $24.8(4.5)$ \\
\hline Normal, no. (\%) & $4709(61.8)$ \\
\hline Overweight, no. (\%) & 1979(26.0) \\
\hline Obese, no. (\%) & $932(12.2)$ \\
\hline Education, no. higher education (\%) & $3055(42.9)$ \\
\hline \multicolumn{2}{|l|}{ Race/ethnicity, no. (\%) } \\
\hline Dutch or European, no. (\%) & $4289(58.2)$ \\
\hline Surinamese, no. (\%) & $655(8.9)$ \\
\hline Turkish, no. (\%) & 673(9.1) \\
\hline Moroccan, no. (\%) & $473(6.4)$ \\
\hline Cape Verdian or Dutch Antilles, no. (\%) & $560(7.6)$ \\
\hline Parity, no. nulliparous (\%) & 4308(56.6) \\
\hline \multicolumn{2}{|l|}{ Smoking, no. (\%) } \\
\hline None, no. (\%) & $4967(72.8)$ \\
\hline Early pregnancy only, no. (\%) & $595(8.7)$ \\
\hline Continued, no. (\%) & $1261(18.5)$ \\
\hline \multicolumn{2}{|l|}{ Birth characteristics } \\
\hline Males, no. (\%) & $3861(50.3 \%)$ \\
\hline Gestational age, median (IQR), weeks & 40.1 (39.1 to 41.0$)$ \\
\hline Birth weight, mean (SD) grams & $3423(544)$ \\
\hline Preterm birth ${ }^{2}$, no. (\%) & $345(4.5)$ \\
\hline Spontaneous preterm birth, no. (\%) & 294(3.0) \\
\hline $\begin{array}{l}\text { Small-size for gestational age } \mathrm{a}^{3}<10 \text { birth centile } \\
(<-1.4 \mathrm{SDS}) \text {, no. }(\%)\end{array}$ & $768(10)$ \\
\hline $\begin{array}{l}\text { Large-size for gestational age }{ }^{3}>90 \text { birth centile } \\
(>1.18 \mathrm{SDS}), \text { no. }(\%)\end{array}$ & $767(10)$ \\
\hline Cesarean delivery, no. (\%) & $836(11.9)$ \\
\hline Assisted vaginal delivery, no. (\%) & $964(13.8)$ \\
\hline Apgar score below 7 at 5 min, no. (\%) & $78(1.0)$ \\
\hline
\end{tabular}

${ }^{a}$ Values are observed data and represent means (SD), medians (IQR) or number of subjects (valid \%). Abbreviations: IQR inter quartile range, $S D$ standard deviation

${ }^{1}$ Body mass index is defined as normal (BMI < 25), overweight (BMI 25-30), obese $(\mathrm{BMI}>30)$

${ }^{2}$ Preterm birth is defined as birth $<37$ weeks of gestational age ${ }^{3} \mathrm{SGA}$ is defined as $<10$ th percentile of gestational age- and sex-adjusted birth weight; LGA is defined as > 90th percentile of gestational age- and sexadjusted birth weight 
placental characteristics. Non-response analyses showed that women without placental measurements were more likely to have a higher BMI and lower educational level (Additional file 1, Table S2). Of all newborns with a second trimester EFW < 10th percentile or $>90$ th percentile, 91 (5.9\%) were born preterm, 214 (13.9\%) were born SGA and 179 (11.7\%) were born LGA. Of all newborns with a third trimester EFW < 10th or $>90$ th percentile, $110(7.2 \%)$ were born preterm, $335(21.8 \%)$ were born SGA and 277 (18.1\%) were born LGA (Table 2). In univariate logistic regression analyses, all maternal exposures were associated with at least one of the adverse birth outcomes, whereas EFW was associated with all three adverse birth outcomes (results available upon request).

\section{Screening for risks of preterm birth}

Figure 1 shows that the maternal characteristics model had a moderate performance for the detection of preterm birth (AUC 0.60 (95\% CI 0.57 to 0.63), which did not improve by adding second trimester EFW (AUC 0.61 ( $95 \%$ CI 0.58 to 0.64 )). Screening improved by adding third trimester EFW (AUC 0.64 (95\% CI 0.61 to $0.67)$ to the maternal characteristics model ( $p$ value for AUC comparison to the maternal characteristics model $<0.01$, Additional file 1, Table S3). AUC effect estimates did not further improve by combining second and third trimester EFW results or using EFW change. Adding placenta flow measures to the third trimester EFW model strongly improved detection of preterm birth (AUC of 0.72 (95\% CI 0.66 to 0.77), $p$ value for model comparison to the third trimester EFW model $<0.01$, Additional file 1, Table S3). Compared to the second trimester model, the third trimester model with placenta flow measures nearly doubled detection of fetuses at risk of preterm birth, as sensitivity increases from 19\% for the second trimester model to $38 \%$ for the third trimester model with placenta flow measures (PLR 3.8; NLR 0.69; PPV 15\%; NPV: 97\%) at 90\% specificity (Fig. 1, Additional file 1, Table S4).

We observed similar model performances when we only took spontaneous preterm birth into account (Additional file 1, Figure S2). Using stricter diagnostic cut-offs led to similar AUCs and sensitivities (Fig. 1, Additional file 1, Figure S3). We did not observe differences in results when we used AC instead of EFW (Additional file 1, Figure S4). Overall, combined use of UtA-RI and UA-PI tended to be better than separate use (Additional file 1, Figure S5). Additional file 1, Figure S6 shows that without maternal characteristics, screening performance of the third trimester model with placenta flow measures for preterm birth was considerably lower.

\section{Screening for risks of small size and large size for gestational age at birth}

The maternal characteristics model and second trimester model had a moderate screening performance for detection of SGA at birth (AUCs 0.67 (95\% CI 0.65 to 0.69$)$ and 0.72 (95\% CI 0.70 to 0.74 ), respectively) (Fig. 2). Compared to these models, the third trimester model significantly improved detection (AUC 0.79 (95\% CI 0.78 to 0.81 ) with a sensitivity of $50 \%$ at $90 \%$ specificity ( $p$ value for AUC

Table 2 Adverse birth outcomes by second and third trimester estimated fetal weight screening results $(N=7670)^{a}$

\begin{tabular}{|c|c|c|c|c|c|c|c|c|c|}
\hline & \multicolumn{3}{|c|}{ Preterm birth } & \multicolumn{3}{|c|}{$\begin{array}{l}\text { Small size for gestational age at } \\
\text { birth }\end{array}$} & \multicolumn{3}{|c|}{$\begin{array}{l}\text { Large size for gestational age at } \\
\text { birth }\end{array}$} \\
\hline & Yes & No & Total & Yes & No & Total & Yes & No & Total \\
\hline \multicolumn{10}{|l|}{ 2nd trimester } \\
\hline $\begin{array}{l}\text { Estimated fetal weight }<10 \text { th percentile } \\
\text { (screen-positive) }\end{array}$ & $41(5.3 \%)$ & 726 (94.7\%) & 767 & $192(25.0 \%)$ & 575 (75.0\%) & 767 & 30 (3.9\%) & 737 (96.1\%) & 767 \\
\hline $\begin{array}{l}\text { Estimated fetal weight 10-90th percentile } \\
\text { (screen negative) }\end{array}$ & $254(4.1 \%)$ & 5882 (95.9\%) & 6136 & 554 (9.0\%) & 5582 (91.0\%) & 6136 & $588(9.6 \%)$ & 5548 (90.4\%) & 6136 \\
\hline $\begin{array}{l}\text { Estimated fetal weight }>90 \text { th percentile } \\
\text { (screen-positive) }\end{array}$ & $50(6.5 \%)$ & 717 (93.5) & 767 & $22(2.9 \%)$ & 745 (97.1\%) & 767 & 149 (19.4\%) & $618(80.6 \%)$ & 767 \\
\hline Total & 345 & 7325 & 7670 & 768 & 6902 & 7670 & 767 & 6903 & 7670 \\
\hline 3rd trimester & Yes & No & Total & Yes & No & Total & Yes & No & Total \\
\hline $\begin{array}{l}\text { Estimated fetal weight }<10 \text { th percentile } \\
\text { (screen-positive) }\end{array}$ & 75 (9.8\%) & 692 (90.2\%) & 767 & $331(43.2 \%)$ & 436 (58\%) & 767 & $4(0.5 \%)$ & 763 (99.5\%) & 767 \\
\hline $\begin{array}{l}\text { Estimated fetal weight 10-90th percentile } \\
\text { (screen negative) }\end{array}$ & $235(3.8 \%)$ & 5901 (96.2\%) & 6136 & 433 (7.1\%) & 5703 (92.9\%) & 6136 & $490(8 \%)$ & $5646(92 \%)$ & 6136 \\
\hline $\begin{array}{l}\text { Estimated fetal weight }>90 \text { th percentile } \\
\text { (screen-positive) }\end{array}$ & $35(4.6 \%)$ & 732 (95.4\%) & 767 & $4(0.5 \%)$ & 763 (99.5\%) & 767 & $273(35.6 \%)$ & 494 (64.4\%) & 767 \\
\hline Total & 345 & 7325 & 7670 & 768 & 6902 & 7670 & 767 & 6903 & 7670 \\
\hline
\end{tabular}

${ }^{\mathrm{a} V a l u e s}$ are absolute numbers (\% of total within the corresponding screening category) 
Screening performance for preterm birth ${ }^{\mathrm{a}}$

$\begin{array}{cc}\begin{array}{c}\text { Screening performance for preterm birth based on maternal characteristics } \\ \text { and estimated fetal weight }{ }^{\mathrm{b}}\end{array} & \begin{array}{c}\text { Screening performance for preterm birth based on maternal characteristics, } \\ \text { estimated fetal weight, and placenta flow measures }\end{array}\end{array}$

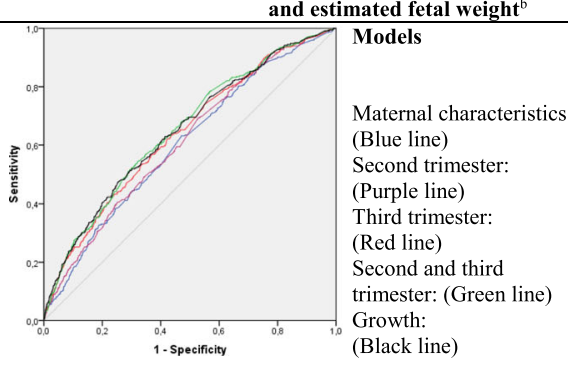

AUC
$\begin{gathered}\text { (95\% Confidence } \\ \text { Interval) }\end{gathered}$
$0.60(0.57$ to 0.63$)$
$0.61(0.58$ to 0.64$)$
$0.64(0.61$ to 0.67$)$
$0.65(0.62$ to 0.68$)$
$0.65(0.62$ to 0.68$)$

1.0

Maternal characteristics:

$\%$ Confidence

Interval)

(Blue line)

Second trimester:

(Purple line)

Third trimester:

(Red line)

$0.68(0.62$ to 0.73$)$

Second and third $\quad 0.75(0.69$ to 0.80$)$

trimester: (Green line)

$0.65(0.62$ to 0.68$)$

,. Growth:

(Black line)

\begin{tabular}{|c|c|c|c|c|c|}
\hline \multicolumn{3}{|c|}{$\begin{array}{l}\text { Sensitivity of the third-trimester-models based on estimated fetal weight } \\
\text { at different diagnostic thresholds and specificities }{ }^{d}\end{array}$} & \multicolumn{3}{|c|}{$\begin{array}{l}\text { Sensitivity of the third-trimester-models based on estimated fetal weight and } \\
\text { placenta flow measures at different diagnostic thresholds and specificities }\end{array}$} \\
\hline & $\begin{array}{c}\text { Estimated fetal weight percentile } \\
\text { diagnostic threshold on } \\
<\text { p10 or }>\text { p90 }\end{array}$ & $\begin{array}{c}\text { Estimated fetal weight percentile } \\
\text { diagnostic threshold on } \\
<\mathrm{p} 5 \text { or }>\mathrm{p} 95\end{array}$ & & $\begin{array}{c}\text { Estimated fetal weight percentile } \\
\text { diagnostic threshold on } \\
<\mathrm{p} 10 \text { or }>\text { p } 90\end{array}$ & $\begin{array}{c}\text { Estimated fetal weight percentile } \\
\text { diagnostic threshold on } \\
<\mathrm{p} 5 \text { or }>\mathrm{p} 95\end{array}$ \\
\hline Specificity & & & Specificity & & \\
\hline $70 \%$ & $48 \%$ & $49 \%$ & $70 \%$ & $61 \%$ & $54 \%$ \\
\hline $80 \%$ & $37 \%$ & $38 \%$ & $80 \%$ & $52 \%$ & $46 \%$ \\
\hline $90 \%$ & $24 \%$ & $25 \%$ & $90 \%$ & $38 \%$ & $34 \%$ \\
\hline
\end{tabular}

AUC: area under the curve; CI: Confidence interval; EFW: Estimated fetal weight;

${ }^{\text {a }}$ Receiver operating characteristic curve for the detection of fetuses at risk for preterm birth based on second and third trimester fetal ultrasound and placenta measures, and derived sensitivity and specificity of the third-trimester-model.

${ }^{b}$ Model characteristics: Maternal characteristics; Second-trimester-model: Maternal characteristics and 2 ${ }^{\text {nd }}$ trimester EFW; Third-trimester-model: Maternal

characteristics and $3^{\text {rd }}$ trimester EFW; Combined model: Maternal characteristics, $2^{\text {nd }}$ and $3^{\text {rd }}$ trimester EFW; Growth model: Maternal characteristics, $2^{\text {nd }}$ and

$3^{\text {rd }}$ trimester EFW and $2^{\text {nd }}$ to $3^{\text {rd }}$ trimester EFW change.

${ }^{c}$ AUCs based on a sample of 2705 participants with all placenta measures available. EFW models as described under ${ }^{b}$ with additional placenta measures.

Second-trimester-model: $2^{\text {nd }}$ trimester UtA-RI and $2^{\text {nd }}$ trimester UA-PI. Third-trimester-model; $3^{\text {rd }}$ trimester UtA-RI and $3^{\text {rd }}$ trimester UA-PI; Second and

third-trimester-model: $2^{\text {nd }}$ and $3^{\text {rd }}$ trimester UtA-RI, and $2^{\text {nd }}$ and $3^{\text {rd }}$ trimester UA-PI; Growth model: $2^{\text {nd }}$ and $3^{\text {rd }}$ trimester UtA-RI and $2^{\text {nd }}$ and $3^{\text {rd }}$ trimester UA-

$\mathrm{PI}, 2^{\text {nd }}$ to $3^{\text {rd }}$ trimester change of UtA-RI and UA-PI.

${ }^{\mathrm{d}}$ Effects of changing the diagnostic threshold on the sensitivity of the third-trimester-model used for screening of preterm birth.

Fig. 1 Screening performance for preterm birth

comparison to the maternal characteristics model and second trimester model $<0.01$, Additional file 1 , Table S3). Compared to the second trimester model, the third trimester model increased detection of fetuses at risk of SGA by a third, as sensitivity increases from $33 \%$ for the second trimester model to $50 \%$ for the third trimester model at $90 \%$ specificity (Fig. 2, Additional file 1, Table S4). Effect estimates of the AUCs did not further clinically improve by combining second and third trimester EFW results or using EFW change. Adding placenta flow measures to the third trimester model did slightly improve screening performance for SGA at birth (AUC 0.83 (95\% CI 0.81 to 0.86) $p$ value for AUC comparison to the third trimester model < 0.01, Fig. 2, Additional file 1, Table S3) leading to a sensitivity of $55 \%$ at $90 \%$ specificity (PLR 5.5; NLR 0.5; PPV 38\%; NPV 95\%). The third trimester model had the best screening performance for detecting LGA with an AUC of 0.76 (95\% CI 0.75 to 0.78 ) and corresponding sensitivity of $43 \%$ at $90 \%$ specificity (Fig. 3). Compared to the second trimester model, the third trimester model increased the detection of fetuses at risk of LGA by a third, as the sensitivity increases from $28 \%$ for the second trimester model to $43 \%$ for the third trimester model (PLR 4.3; NLR 0.63; PPV 32\%; NPV 93\%) at 90\% specificity (Fig. 3, Additional file 1, Table S4). Adding placenta flow measures to the third trimester model did not improve LGA screening performance.
Model performance was the same when screenpositive was defined separately for SGA and LGA, as when screen-positive was defined as one screening test for both SGA and LGA (Additional file 1, Figure S7). When we used stricter diagnostic cut-offs (screen-positive defined as EFW $<5$ th or $>95$ th percentile), the sensitivities for detection of SGA and LGA slightly decreased (Figs. 2 and 3 respectively, ROCs and AUCs in Additional file 1, Figure S3). When we used stricter outcome cut-offs (extreme SGA and LGA defined as gestational-age-adjusted birth weight $<3$ rd or $>97$ th percentile, and moderate SGA or LGA defined as gestational-age-adjusted birth weight $<5$ th or $>95$ th percentile, respectively), the model performance slightly improved as compared to our main analysis (sensitivities, ROCs and AUCs in Additional file 1, Figure S8 and Figure S9). When we assessed screening performance for SGA newborns with pregnancies complicated by gestational hypertensive disorders and LGA newborns with pregnancies resulting in assisted vaginal delivery or cesarean section, we observed similar model performance as our main analysis (Additional file 1, Figure S10). We did not observe differences in results when we used AC instead of EFW (Additional file 1, Figure S4). When we excluded maternal smoking from the models, results were similar (findings not shown). Without incorporating maternal characteristics in the screening models, 


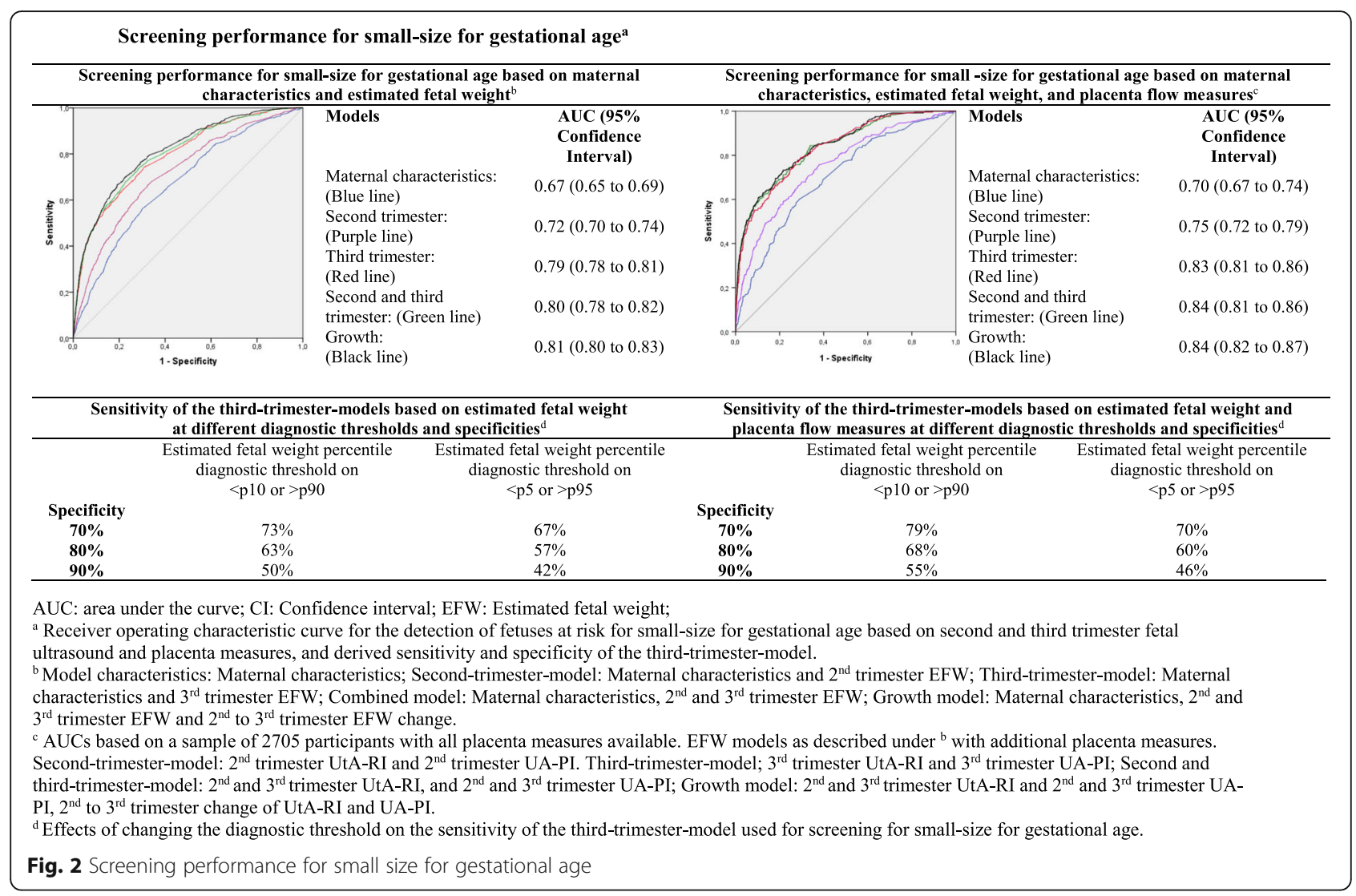

screening performance of the third trimester model for SGA and LGA was considerably lower (Additional file 1, Figure S6).

\section{Discussion}

Our results suggest that third trimester ultrasound examination in addition to maternal characteristics has the best screening performance for detecting fetuses at risk for preterm birth, SGA and LGA, compared to second trimester ultrasound or combined second and third trimester ultrasound. Compared to second trimester ultrasound screening, third trimester ultrasound screening would nearly double detection of fetuses at risk of these adverse birth outcomes in a low-risk population.

\section{Interpretation of main findings}

Preterm birth, SGA and LGA are strongly related to perinatal morbidity and mortality and have long-term consequences for disease risk [2, 4, 33]. Abnormal fetal growth and impaired placental function are important risk factors for adverse birth outcomes, with the strongest associations observed for third trimester fetal and placental measures $[3-5,29]$. Despite these observed associations, the additional clinical value for third trimester screening for fetuses at risk for common adverse birth outcomes remains unclear. A review of 13 controlled trials showed no beneficial effects of routinely performed ultrasound after 24 weeks GA on pregnancy outcomes [13]. These trials were mainly performed in the early 1990s. Recent developments in ultrasound techniques and treatment protocols, and changes in prevalence of women at risk of abnormal fetal growth limit the applicability of these results to current clinical practice. Technological ultrasound advancements in obstetrics may lead to implementation of fetal size and anomaly scans in first trimester and fetal growth assessment later in pregnancy. Further insight into the optimal period for ultrasound screening for adverse birth outcomes is therefore urgently needed.

Despite reported associations of suboptimal fetal growth and impaired placental function with preterm birth, no previous studies assessed the screening performance of second and third trimester ultrasound for preterm birth risk [3, 34]. We observed that third trimester fetal and placental ultrasound together with maternal characteristics had the best screening performance for preterm birth. We did not find a benefit of second to third trimester EFW change for screening for preterm birth, although previously published work from our cohort showed that second to third trimester EFW change was associated with the risk of preterm birth [3]. In this previous analysis, we only assessed the association of second to third trimester 


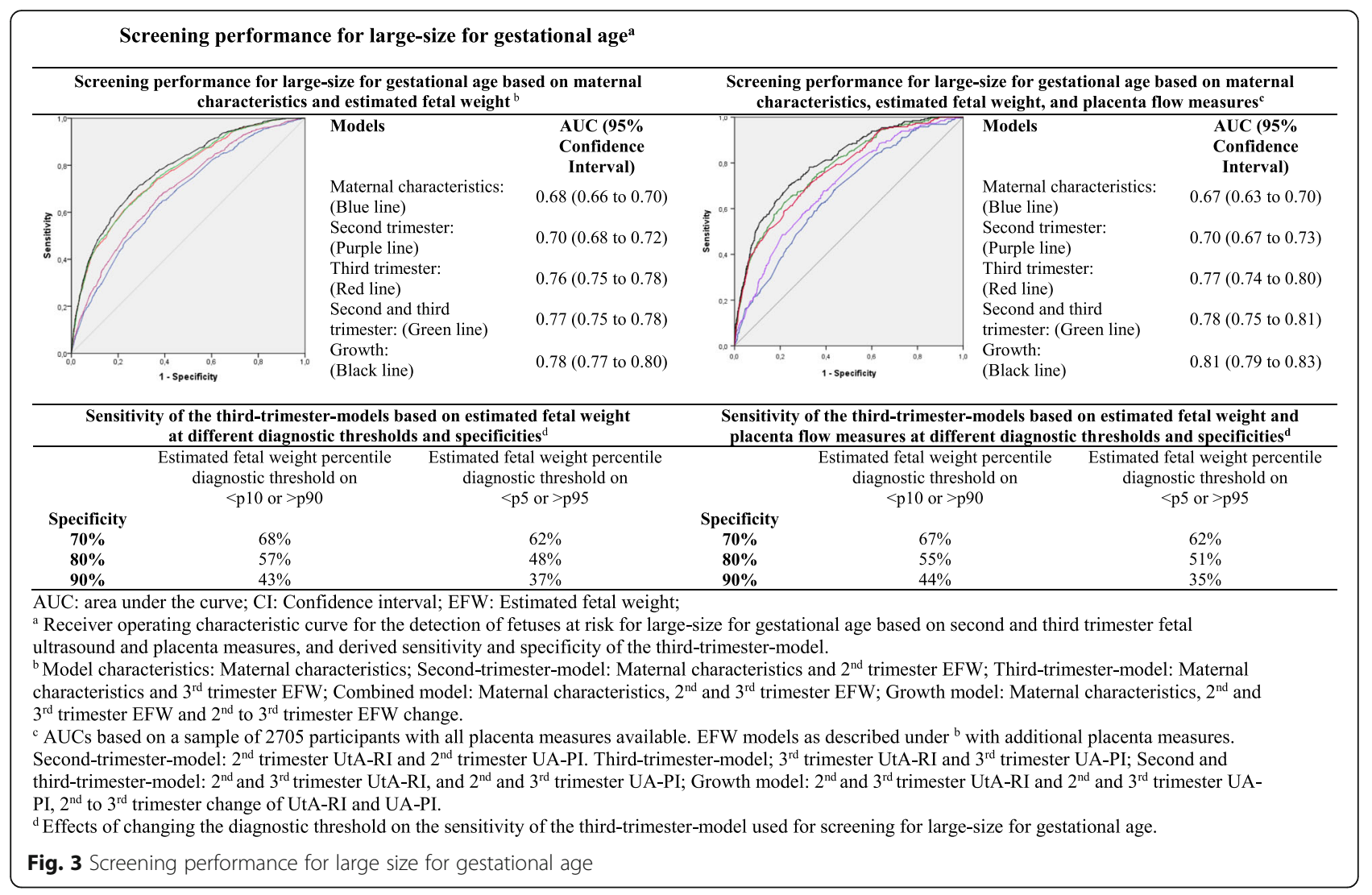

EFW change with the risk of preterm birth and did not consider second or third trimester fetal size in the analysis. Contrary, in our current analysis, we assessed the screening performance for preterm birth of the addition of second to third trimester EFW change to second and third trimester fetal size, and observed it did not further improve screening performance. Thus, it seems that an association between second to third trimester EFW change with the risk of preterm birth is present, but that this does not add to screening performance for preterm birth when we also consider second and third trimester fetal size. The additional value of placenta measures to the screening model may be explained by the role of placental dysfunction in preterm birth [1]. We observed the strongest screening performance for using a combination of umbilical and uterine artery resistance indices. However, differences compared to single use of either measurement were small. As the umbilical artery pulsatility indices are technically easier to measure, this measure might be most appropriate for use in clinical practice. Overall, in our relatively healthy low-risk population, the combination of third trimester fetal and placental ultrasound with maternal characteristics led to a doubling of antenatally identified newborns at risk for preterm birth compared to second trimester ultrasound or maternal characteristics only. A limited number of previous studies explored screening performance by single and combined second and third trimester EFW or AC measurements for prediction of SGA or LGA, taking into account maternal characteristics. A retrospective study among 3520 women reported a moderate screening performance for SGA with a sensitivity of $41.8 \%$ at $90 \%$ specificity using a combination of maternal factors, first trimester chemistry results and second trimester EFW and placenta measures [35]. Another retrospective cohort study among 1979 women reported that adding maternal characteristics and third trimester fetal and placental ultrasound to second trimester ultrasound results improved sensitivity from 51.3 to $69.7 \%$ for SGA and from 44.1 to $59.4 \%$ for LGA at $90 \%$ specificity [36]. A recent cohort study among 3440 pregnancies assessed the screening value of single versus serial fetal biometry at 28, 32 and 36 weeks GA for SGA and LGA [37]. This study observed that single fetal biometry at 32 weeks had a higher sensitivity than longitudinal analysis from more observations projecting EFW at 40 weeks [37]. In our study, the third trimester ultrasound was performed at an average of 30 weeks of gestation, as compared to an average 34 to 36 weeks of gestation in other studies [37, 38]. Although screening performance of third trimester ultrasound may improve when performed later in the third trimester, third 
trimester ultrasound screening around 30 weeks' gestation is valuable as it offers a larger window for interventions. In our study, we observed that third trimester fetal and placental ultrasound together with maternal characteristics had the best screening performance for SGA and LGA. Already in our low-risk population, this approach led to a third increase in detection of fetuses at risk of SGA or LGA compared to second trimester ultrasound screening. We did not observe additional screening benefit for combining second and third trimester ultrasounds or for using AC instead of EFW.

It is well-established that newborns born SGA or LGA may be both constitutionally or pathologically small or large for their gestational age [39]. It has been suggested that newborns who are pathologically small or large for their gestational age due to abnormal fetal growth have increased risks of morbidity and mortality, as compared to those newborns who are constitutionally small or large for their gestational age [39]. To better distinguish potential pathological SGA and LGA newborns from constitutional SGA and LGA newborns, we also assessed the screening performance of our screening models for moderate and extreme SGA and LGA, and for SGA and LGA complicated by pregnancy or delivery complications. We found that the screening performance was similar. This suggests our third trimester screening model may aid in the identification of newborns who are pathologically small or large for their gestational age. We did not use customized birth weight centiles for classification of abnormal size at birth as a method to distinguish potential pathological SGA and LGA newborns from constitutional SGA or LGA newborns, as previous studies have not shown strong results regarding the use of customized charts to identify SGA or LGA newborns at higher risk of mortality and adverse short-term and long-term outcomes [40, 41]. A limitation of our cohort is that we do not have extensive information available on neonatal morbidity. Further studies are needed to replicate our findings and to assess whether our screening model identifies SGA and LGA born newborns at risk of morbidity and mortality, considering more detailed measures of neonatal morbidity.

Overall, we observed slightly lower sensitivities for screening for SGA and LGA than previous studies, which could be explained by taking into account maternal characteristics, our relatively healthy low-risk population and the earlier timing of third trimester ultrasound $[37,38]$. As maternal characteristics are simple and cost-effective measurements, easily available within clinical practice, we specifically aimed to assess their screening performance for screening of adverse birth outcomes within low-risk populations and the subsequent additional screening performance of more expensive and time-consuming fetal and placental ultrasound measurements. We found that in absence of maternal characteristics, the screening models had an inferior screening performance compared to when maternal characteristics were taken into account but the third trimester fetal and placental ultrasound still had the best screening performance for adverse birth outcomes. Thus, our findings underline the importance of considering maternal characteristics within low-risk populations for screening of adverse birth outcomes and the potential value of third trimester ultrasound.

Our findings suggest that implementation of third trimester fetal and placental ultrasound, combined with common maternal characteristics, would nearly double detection of fetuses at risk for common adverse birth outcomes compared to second trimester ultrasound and provides further evidence for critical evaluation of current obstetric care guidelines. Improved detection of fetuses at risk of preterm birth, SGA and LGA provides the clinician the opportunity to optimize monitoring and interventions [42]. Monitoring could be intensified by additional assessments of fetal size, cervical length and umbilical artery waveforms using (Doppler) ultrasound and fetal wellbeing using cardiotocography, which might further improve detection of fetuses at risk of adverse outcomes whom may benefit from interventions, such as administering steroids for fetal lung maturation if preterm birth is imminent or termination of pregnancy if signs of placental insufficiency occur. Previous studies have shown that SGA or LGA newborns who were identified antenatally have lower risks of morbidity and mortality, compared to those who were unidentified antenatally [6-9]. However, it has also been suggested that prenatal diagnosis of abnormal fetal growth may lead to poorer outcomes due to subsequent interventions [43]. Benefits due to identification of true positives versus harm caused by false positives and interventions should be evaluated. Future welldesigned randomized controlled trials are needed to confirm our results and to assess whether the advantages of screening outweigh the potential harm from parental anxiety and iatrogenic morbidity, in contemporary low-risk populations.

\section{Strengths and limitations}

We had a prospective data collection from early pregnancy onwards and a large sample of 7670 women with fetal growth measurements available. The nonresponse at baseline might have led to selection of a more healthy population, which might affect the generalizability of results to high-risk populations. We also had a relatively small number of cases of adverse 
birth outcomes, which might further indicate a selection towards a low-risk population. To assess whether a screening model improved by adding additional maternal, fetal or placental characteristics, we assessed if changes in AUC effect estimates of different screening models were clinically relevant and whether the differences in AUCs of two different models were statistically significant. What is considered clinically relevant may be arbitrary. Based on previous studies focused on screening for similar adverse birth outcomes, we considered an approximate $4-5 \%$ change in effect estimate of the AUC as clinically relevant, as this change is likely associated with a detectable increase in sensitivity $[14,28,44]$. Next, when model comparison fulfilled this criterion, we used a statistical test by DeLong et al. to see if this change was statistically significant [32]. This method takes into account two correlated AUCs, which is necessary as two curves are constructed based on the same individuals. We included common maternal characteristics, easily available within all pregnant women and applicable to low-risk pregnant populations, within our maternal screening model. Another predictor for preterm birth, SGA or LGA at birth is occurrence of either of these outcomes in a previous pregnancy. We did not use this maternal characteristic for screening in our models, as women with a previous preterm birth, SGA or LGA newborn are already considered higher risk pregnant women and often intensified monitoring and additional ultrasounds for fetal growth are indicated. Among higherrisk populations, a different third trimester ultrasound screening model including other maternal characteristics may be more applicable or even a separate screening model for nulliparous and multiparous women may be needed. Further studies assessing screening performance for adverse birth outcomes of third trimester fetal and placental ultrasound, in combination with more maternal characteristics such as previous pregnancy complications, among high-risker populations are needed. All ultrasound measurements were performed according to the study protocol and blinded with regard to pregnancy outcomes due to the prospective nature of the study. Abnormal research ultrasound results were reported to healthcare providers and some participants might have been treated as a consequence of abnormal (research) ultrasound findings, which might have affected the screening performance. For example, if an abnormal EFW in a research ultrasound was found, this may have led to induction of labour before 37 weeks of gestation, which is considered iatrogenic preterm birth. However, when we restricted our analyses to spontaneous preterm birth only, we found similar screening performance. Thus, the performance of our model screening for preterm birth does not seem to be driven by iatrogenic preterm birth.

\section{Conclusion}

Maternal characteristics together with single third trimester fetal and placental ultrasound has the best screening performance for preterm birth, and SGA and LGA at birth, compared to using only second trimester ultrasound or combined second and third trimester ultrasound. Compared to second trimester ultrasound screening, third trimester ultrasound screening would nearly double detection of fetuses at risk of these common adverse birth outcomes in low-risk populations.

\section{Supplementary information}

The online version of this article (https://doi.org/10.1186/s12916-020-01540-x) contains supplementary material, which is available to authorized users.

Additional file 1 Index supplemental material.

Additional file $\mathbf{2}$ STROBE checklist.

\section{Acknowledgements}

The Generation R Study is conducted by the Erasmus Medical Center in close collaboration with the School of Law and Faculty of Social Sciences of the Erasmus University Rotterdam, the Municipal Health Service Rotterdam area, Rotterdam, the Rotterdam Homecare Foundation, Rotterdam, and the Stichting Trombosedienst and Artsenlaboratorium Rijnmond (STAR). We gratefully acknowledge the contribution of participating mothers, general practitioners, hospitals, midwives and pharmacies in Rotterdam.

\section{Funding}

The Generation R Study was supported by financial support by the Erasmus University Medical Center, Rotterdam, the Erasmus University Rotterdam, the Netherlands Organization for Health Research and Development (ZonMw), the Netherlands Organization for Scientific Research (NWO), the Ministry of Health, Welfare and Sport. This study was supported by a grant from the Netherlands Organization for Health Research and Development Pregnancy \& Birth Programme (543003109). Vincent Jaddoe received a grant from the European Research Council (Consolidator Grant, ERC-2014-CoG-648916). Romy Gaillard received funding from the Dutch Heart Foundation (grant number 2017 T013), the Dutch Diabetes Foundation (grant number 2017.81.002) and ZonMw (grant number 543003109).

Availability of data and materials

Data requests can be made to the secretariat of Generation $R$.

Authors' contributions

$J E, V J$ and RG designed the study and performed its implementation, designed the study's analytical strategy and performed the analyses and preparation of the text. EV, AM, ES, LD and IR advised and reviewed the manuscript for important intellectual content. All authors read and approved the final manuscript.

Ethics approval and consent to participate

The study has been approved by the local Medical Ethical Committee (MEC 198.782/2001/31). Written consent was obtained from all participating women.

Consent for publication

Not applicable.

\section{Competing interests}

The authors declare that they have no competing interests.

\section{Author details}

The Generation R Study Group, Erasmus MC, University Medical Center Rotterdam, P.O. Box 2040, 3000, CA, Rotterdam, the Netherlands.

${ }^{2}$ Department of Paediatrics, Erasmus MC, University Medical Center

Rotterdam, Rotterdam, The Netherlands. ${ }^{3}$ Department of Obstetrics and 
Gynaecology, Erasmus MC, University Medical Center Rotterdam, Rotterdam, The Netherlands. ${ }^{4}$ Department of Paediatrics, Division of Neonatology, Erasmus MC, University Medical Center Rotterdam, Rotterdam, The Netherlands. ${ }^{5}$ Department of Paediatrics, Division of Respiratory Medicine and Allergology, Erasmus MC, University Medical Center Rotterdam, Rotterdam, The Netherlands.

Received: 30 September 2019 Accepted: 20 February 2020 Published online: 07 April 2020

\section{References}

1. Simmons LE, Rubens CE, Darmstadt GL, Gravett MG. Preventing preterm birth and neonatal mortality: exploring the epidemiology, causes, and interventions. Semin Perinatol. 2010;34(6):408-15.

2. Katz J, Lee AC, Kozuki N, Lawn JE, Cousens S, Blencowe H, Ezzati M, Bhutta ZA, Marchant T, Willey BA, et al. Mortality risk in preterm and small-forgestational-age infants in low-income and middle-income countries: a pooled country analysis. Lancet. 2013;382(9890):417-25.

3. Gaillard R, Steegers EA, de Jongste JC, Hofman A, Jaddoe WW. Tracking of fetal growth characteristics during different trimesters and the risks of adverse birth outcomes. Int J Epidemiol. 2014;43(4):1140-53.

4. Pallotto EK, Kilbride HW. Perinatal outcome and later implications of intrauterine growth restriction. Clin Obstet Gynecol. 2006;49(2):257-69.

5. Rosenberg A. The IUGR newborn. Semin Perinatol. 2008;32(3):219-24.

6. Lindqvist PG, Molin J. Does antenatal identification of small-for-gestational age fetuses significantly improve their outcome? Ultrasound Obstet Gynecol. 2005;25(3):258-64.

7. De Reu PA, Oosterbaan HP, Smits $\sqcup$, Nijhuis JG. Avoidable mortality in small-for-gestational-age children in the Netherlands. J Perinat Med. 2010; 38(3):311-8.

8. Boulvain M, Senat MV, Perrotin F, Winer N, Beucher G, Subtil D, Bretelle F, Azria E, Hejaiej D, Vendittelli F, et al. Induction of labour versus expectant management for large-for-date fetuses: a randomised controlled trial. Lancet. 2015;385(9987):2600-5.

9. Smith-Bindman R, Chu PW, Ecker J, Feldstein VA, Filly RA, Bacchetti P. Adverse birth outcomes in relation to prenatal sonographic measurements of fetal size. J Ultrasound Med. 2003;22(4):347-56. quiz 357-348.

10. VanderWeele TJ, Lauderdale DS, Lantos JD. Medically induced preterm birth and the associations between prenatal care and infant mortality. Ann Epidemiol. 2013;23(7):435-40.

11. Press R. Antenatal care: Routine care for the healthy pregnant woman. In: RCOG Press at the Royal College of Obsstetricians and Gynaecologists; 2008.

12. American College of O, Gynecologists. ACOG Practice Bulletin No. 101: ultrasonography in pregnancy. Obstet Gynecol. 2009;113(2 Pt 1):451-61.

13. Bricker L, Medley N, Pratt JJ. Routine ultrasound in late pregnancy (after 24 weeks' gestation). Cochrane Database Syst Rev. 2015;6:CD001451.

14. Sovio U, White IR, Dacey A, Pasupathy D, Smith GCS. Screening for fetal growth restriction with universal third trimester ultrasonography in nulliparous women in the pregnancy outcome prediction (POP) study: a prospective cohort study. Lancet. 2015;386(10008):2089-97.

15. Jaddoe WW, van Duijn CM, Franco OH, van der Heijden AJ, van lizendoorn $\mathrm{MH}$, de Jongste JC, van der Lugt A, Mackenbach JP, Moll HA, Raat H, et al. The generation R study: design and cohort update 2012. Eur J Epidemiol. 2012;27(9):739-56

16. Jaddoe WW, Mackenbach JP, Moll HA, Steegers EAP, Tiemeier H, Verhulst FC, Witteman JCM, Hofman A. The generation R study: design and cohort profile. Eur J Epidemiol. 2006;21(6):475.

17. von Elm E, Altman DG, Egger M, Pocock SJ, Gotzsche PC, Vandenbroucke JP, Initiative S. The Strengthening the Reporting of Observational Studies in Epidemiology (STROBE) statement: guidelines for reporting observational studies. J Clin Epidemiol. 2008;61(4):344-9.

18. Gaillard R, Rurangirwa AA, Williams MA, Hofman A, Mackenbach JP, Franco $\mathrm{OH}$, Steegers EA, Jaddoe WW. Maternal parity, fetal and childhood growth, and cardiometabolic risk factors. Hypertension. 2014;64(2):266-74.

19. Gaillard R, Durmus B, Hofman A, Mackenbach JP, Steegers EA, Jaddoe WW. Risk factors and outcomes of maternal obesity and excessive weight gain during pregnancy. Obesity (Silver Spring). 2013;21(5):1046-55.

20. Bakker R, Steegers EA, Biharie AA, Mackenbach JP, Hofman A, Jaddoe WW. Explaining differences in birth outcomes in relation to maternal age: the generation R study. BJOG. 2011;118(4):500-9.
21. Verburg BO, Steegers EA, De Ridder M, Snijders RJ, Smith E, Hofman A, Moll HA, Jaddoe WW, Witteman JC. New charts for ultrasound dating of pregnancy and assessment of fetal growth: longitudinal data from a population-based cohort study. Ultrasound Obstet Gynecol. 2008;31(4):388-96.

22. Hadlock FP, Harrist RB, Sharman RS, Deter RL, Park SK. Estimation of fetal weight with the use of head, body, and femur measurements--a prospective study. Am J Obstet Gynecol. 1985;151(3):333-7.

23. Di Lorenzo G, Monasta L, Ceccarello M, Cecotti V, D'Ottavio G. Third trimester abdominal circumference, estimated fetal weight and uterine artery doppler for the identification of newborns small and large for gestational age. Eur J Obstet Gynecol Reprod Biol. 2013;166(2):133-8.

24. Nakling J, Backe B. Adverse obstetric outcome in fetuses that are smaller than expected at second trimester routine ultrasound examination. Acta Obstet Gynecol Scand. 2002;81(9):846-51.

25. Figueras F, Gardosi J. Intrauterine growth restriction: new concepts in antenatal surveillance, diagnosis, and management. Am J Obstet Gynecol. 2011;204(4):288-300.

26. Singh T, Leslie K, Bhide A, D'Antonio F, Thilaganathan B. Role of secondtrimester uterine artery Doppler in assessing stillbirth risk. Obstet Gynecol. 2012;119(2 Pt 1):256-61.

27. Alfirevic Z, Stampalija T, Dowswell T. Fetal and umbilical Doppler ultrasound in high-risk pregnancies. Cochrane Database Syst Rev. 2017;6:CD007529.

28. Vieira MC, McCowan LME, Gillett A, Poston L, Fyfe E, Dekker GA, Baker PN, Walker JJ, Kenny LC, Pasupathy D, et al. Clinical, ultrasound and molecular biomarkers for early prediction of large for gestational age infants in nulliparous women: an international prospective cohort study. PLoS One. 2017;12(6):e0178484.

29. Gaillard R, Arends LR, Steegers EA, Hofman A, Jaddoe WW. Second- and third-trimester placental hemodynamics and the risks of pregnancy complications: the Generation R Study. Am J Epidemiol. 2013;177(8):743-54.

30. Gaillard R, Steegers EA, Tiemeier H, Hofman A, Jaddoe WW. Placental vascular dysfunction, fetal and childhood growth, and cardiovascular development: the generation R study. Circulation. 2013;128(20):2202-10.

31. Niklasson A, Ericson A, Fryer JG, Karlberg J, Lawrence C, Karlberg P. An update of the Swedish reference standards for weight, length and head circumference at birth for given gestational age (1977-1981). Acta Paediatr Scand. 1991;80(8-9):756-62.

32. DeLong ER, DeLong DM, Clarke-Pearson DL. Comparing the areas under two or more correlated receiver operating characteristic curves: a nonparametric approach. Biometrics. 1988;44(3):837-45.

33. Gluckman PD, Hanson MA, Cooper C, Thornburg KL. Effect of in utero and early-life conditions on adult health and disease. N Engl J Med. 2008;359(1): 61-73.

34. Johnsen SL, Wilsgaard T, Rasmussen S, Hanson MA, Godfrey KM, Kiserud T. Fetal size in the second trimester is associated with the duration of pregnancy, small fetuses having longer pregnancies. BMC Pregnancy Childbirth. 2008;8:25.

35. Sotiriadis A, Figueras F, Eleftheriades M, Papaioannou GK, Chorozoglou G, Dinas K, Papantoniou N. First-trimester and combined first- and secondtrimester prediction of small-for-gestational age and late fetal growth restriction. Ultrasound Obstet Gynecol. 2019;53(1):55-61. https://doi.org/10. 1002/uog.19055. Epub 2018 Nov 26.

36. Papastefanou I, Pilalis A, Chrelias C, Kassanos D, Souka AP. Screening for birth weight deviations by second and third trimester ultrasound scan. Prenat Diagn. 2014;34(8):759-64.

37. Tarca AL, Hernandez-Andrade E, Ahn H, Garcia M, Xu Z, Korzeniewski SJ, Saker H, Chaiworapongsa T, Hassan SS, Yeo L, et al. Single and serial fetal biometry to detect preterm and term small- and large-for-gestational-age neonates: a longitudinal cohort study. PLoS One. 2016;11(11):e0164161.

38. Souka AP, Papastefanou I, Pilalis A, Michalitsi V, Panagopoulos P, Kassanos D. Performance of the ultrasound examination in the early and late third trimester for the prediction of birth weight deviations. Prenat Diagn. 2013; 33(10):915-20.

39. Gaillard R, Jaddoe WW. Assessment of fetal growth by customized growth charts. Ann Nutr Metab. 2014;65(2-3):149-55.

40. Chiossi G, Pedroza C, Costantine MM, Truong VTT, Gargano G, Saade GR. Customized vs population-based growth charts to identify neonates at risk of adverse outcome: systematic review and Bayesian meta-analysis of observational studies. Ultrasound Obstet Gynecol. 2017;50(2):156-66.

41. Erkamp JS, Jaddoe WWV, Mulders A, Steegers EAP, Reiss IKM, Duijts L, Gaillard R. Customized versus population birth weight charts for 
identification of newborns at risk of long-term adverse cardio-metabolic and respiratory outcomes: a population-based prospective cohort study. BMC Med. 2019;17(1):186.

42. Gynecologists RCoOa: The Investigation and Management of the Small-forGestational-Age Fetus: Green-top Guideline No.31. RCOG Press at the Royal College of Obsstetricians and Gynaecologists 2013.

43. Monier I, Blondel B, Ego A, Kaminiski M, Goffinet F, Zeitlin J. Poor effectiveness of antenatal detection of fetal growth restriction and consequences for obstetric management and neonatal outcomes: a French national study. BJOG. 2015;122(4):518-27.

44. Apfel CC, Kranke P, Greim CA, Roewer N. What can be expected from risk scores for predicting postoperative nausea and vomiting? Br J Anaesth. 2001;86(6):822-7.

\section{Publisher's Note}

Springer Nature remains neutral with regard to jurisdictional claims in published maps and institutional affiliations.

Ready to submit your research? Choose BMC and benefit from:

- fast, convenient online submission

- thorough peer review by experienced researchers in your field

- rapid publication on acceptance

- support for research data, including large and complex data types

- gold Open Access which fosters wider collaboration and increased citations

- maximum visibility for your research: over $100 \mathrm{M}$ website views per year

At BMC, research is always in progress.

Learn more biomedcentral.com/submissions 